\title{
Diagnostic Value of D-Dimer in Acute Myocardial Infarction Among Patients With Suspected Acute Coronary Syndrome
}

\author{
Hamidreza Reihani ${ }^{\mathrm{a}}$, Alireza Sepehri Shamloo ${ }^{\mathrm{b}}$, \\ Assadollah Keshmiric, d
}

\begin{abstract}
Background: The role of D-dimer as a diagnostic marker in myocardial infarction (MI) and acute coronary syndrome (ACS) is still a question. The aim of this study was to evaluate the diagnostic value of D-dimer in the diagnosis of AMI in patients suspected with ACS.
\end{abstract}

Methods: This cross-sectional study was conducted on patients suspected with ACS. Serial standard 12-lead electrocardiogram (ECG), D-dimer, and troponin tests were done for all the patients. According to the examinations, ECG changes, and troponin, patients were allocated into two groups of MI and unstable angina (UA). Chi-square, independent $t$-test, and Pearson correlation test were used by SPSS ver, 17. Cut-off point of D-dimer for MI diagnosis was evaluated by receiver operating characteristic (ROC) curve analysis.

Results: Seventy-five patients with a mean age of $63.1 \pm 9.75$ years were studied in two groups of MI $(\mathrm{n}=34)$ and UA $(\mathrm{n}=$ 41). Patients were homogeneous based on age, gender, and risk factors for diabetes and dyslipidemia. D-dimer in patients with MI patients was higher than in patients with UA $(\mathrm{P}=0.001)$. The optimal cut-off point of D-dimer for diagnosis of MI was $548 \mathrm{mEq} / \mathrm{L}$ with sensitivity and specifity of $63.4 \%$ and $91.2 \%$, respectively.

Conclusions: Based on the results of this study, it seems that the measurement of D-dimer serum level can be appropriate as a marker with high sensitivity and relatively high specificity for differentiating MI from UA in patients with suspected ACS.

Keywords: Myocardial infarction; Unstable angina; Acute coronary

Manuscript submitted October 9, 2017, accepted October 19, 2017

aDepartment of Emergency Medicine, Imam Reza Hospital, Mashhad University of Medical Sciences, Mashhad, Iran

${ }^{b}$ Cardiac Anesthesia Research Center, Faculty of Medicine, Mashhad University of Medical Sciences, Mashhad, Iran

'Faculty of Medicine, Mashhad University of Medical Sciences, Mashhad, Iran

${ }^{\mathrm{d} C o r r e s p o n d i n g ~ A u t h o r: ~ A s s a d o l l a h ~ K e s h m i r i, ~ D e p a r t m e n t ~ o f ~ E m e r g e n c y ~}$ Medicine, Imam Reza Hospital, Edalatian Emergency Center, Mashhad, Iran. Email: asad.keshmiri@yahoo.com

doi: https://doi.org/10.14740/cr620w syndrome; D-dimer; Troponin

\section{Introduction}

Acute coronary syndrome (ACS) is a common cause of presentation in emergency departments (EDs) [1]. Early detection and rapid ruleout of acute myocardial infarction (AMI) have always been one of the great concerns to reduce mortality, morbidity, and hospitalization costs and avoid doing further diagnostic and unnecessary interventions in low risk patients $[1,2]$. Routinely, the serial electrocardiograms (ECGs), biomarkers, and clinical decision rules have been used for the risk stratifications and diagnosis of ACS [3]. Cardiac enzymes start to rise within hours after onset of symptoms. On the other hand, ECG has not shown to have a reliable sensitivity in early diagnosis of MI $[1,4]$. Thus, attempts continue to find a better way to triage and a more rapid diagnosis of ACS. Coronary artery thrombosis is the cause of acute ischemic syndromes. Several markers involved in the formation and lysis of arterial thrombosis have been identified among which Fibrinogen, plasmin- $\alpha_{2}$ antiplasmin, prothrombin, activated factor VII and $\mathrm{D}$-dimer can be noted. It is anticipated that the levels of these enzymes change with the incidence of coronary artery thrombosis $[1,3,5]$. The $\mathrm{D}$-dimer was used as a diagnostic marker in venous thromboembolism [6], but few studies recently demonstrated the diagnostic value of D-dimer in the diagnosis of MI and ACS [3, 5, 7-10]. This study aimed to evaluate the diagnostic value of D-dimer in the diagnosis of AMI in patients suspected with ACS in an academic ED.

\section{Methods}

\section{Research design and setting}

This cross-sectional study was conducted from April 2015 to February 2016 in the ED of Imam Reza Hospital, and urban academic ED located in Mashhad, Iran. Inclusion criteria were patients with typical chest pain, which defines as a substernal pain, provoked by exertion, or relieved by rest or nitroglycerin-suspected ACS presented to ED with age greater than 18 years old, onset of symptoms less than $24 \mathrm{~h}$. Exclusion criteria were positive family history of cardiovascular disease, use of 
Table 1. Baseline Characteristics of Patients With Myocardial Infarction Compared to Patients With Unstable Angina

\begin{tabular}{llll}
\hline Variables & Myocardial infarction $(\mathbf{n}=\mathbf{3 4})$ & Unstable angina $(\mathbf{n}=\mathbf{4 1})$ & $\mathbf{P}$ \\
\hline Sex, male, $\mathrm{n}(\%)$ & $22(64.7 \%)$ & $19(46.3 \%)$ & 0.162 \\
Age (mean $\pm \mathrm{SD})$ & $63.3 \pm 6.9$ & $63.0 \pm 11.6$ & 0.886 \\
Diabetes, $\mathrm{n}(\%)$ & $22(64.7 \%)$ & $20(47.8 \%)$ & 0.325 \\
Dyslipidemia, $\mathrm{n}(\%)$ & $12(35.2)$ & $13(31.7)$ & 0.808 \\
Blood glucose (mean $\pm \mathrm{SD}), \mathrm{mg} / \mathrm{dL}$ & $247.0 \pm 94.5$ & $209.5 \pm 91.6$ & 0.086 \\
Troponin (mean $\pm \mathrm{SD}), \mathrm{mg} / \mathrm{dL}$ & $0.73 \pm 0.4$ & $0.16 \pm 0.3$ & 0.001 \\
D-dimer $(\mathrm{mean} \pm \mathrm{SD}), \mathrm{mg} / \mathrm{dL}$ & $1,087.6 \pm 395.6$ & $584.6 \pm 444.1$ & 0.001 \\
\hline
\end{tabular}

steroidal and non-steroidal anti-inflammatory drugs, cytotoxic drugs, beta blockers, nitrate and aspirin, anticoagulants such as warfarin and heparin.

\section{Sampling}

\section{Sample size and method}

Patients were studied in purposive random sampling in the two groups with and without MI. The sample size of this study was estimated based on alpha of 0.05 and beta of $20 \%$ and using comparison of two mean sample size formula. Accordingly, at least 28 patients were studied in each of the two groups.

\section{Data collection}

The patients with typical chest pain for ACS and related history and physical examination were enrolled in the study. Their data about age, sex, duration of pain, location of pain, onset of pain, quality of pain, radiation of pain, associated symptoms, and ACS risk factors such as diabetes and dyslipidemia were collected. All patients were subjected to ECG and blood sampling in order to measure serum level of D-dimer and troponin on ED presentation. Then with ECG's findings and troponin serum levels, they were categorized to STEMI, non-STEMI, or UA.

\section{Measurement tool}

\section{D-dimer}

Serum sample was taken to determine D-dimer level at the time of presentation to ED and before receiving heparin. Serum D-dimer level was measured by enzyme-linked immunosorbent assay (ELISA).

\section{Diagnosis of MI}

Patients with the diagnosis of AMI were classified into two groups of STEMI and non-STEMI according to ECG changes.
Other patients under study were placed in the UA group if not diagnosed with MI.

\section{Ethical consideration}

This study was approved by the Ethics Committee of Mashhad University of Medical Sciences (code: 930281) and all patients were informed about the plan and signed written consent form was available.

\section{Statistical analysis}

Demographic and paraclinical data obtained from patients were statistically analyzed using SPSS 17.0 for Windows (SSPS Inc., Chicago, IL, USA). Data were expressed as mean $\pm \mathrm{SD}$ and $\mathrm{P}<0.05$ was considered significant. How to distribute data was initially examined. Then the homogeneity of the groups under comparison (MI and UA) was studied in terms of age, sex and risk factors for diabetes and dyslipidemia. Then to compare quantitative data (D-dimer serum level and troponin) between MI and UA groups, independent $t$-test and to compare the data among the three groups of STEMI, NSTEMI, and UA, ANOVA test were used. In case of non-normal distribution of data, non-parametric equivalents of tests were used. In order to determine cut-off point to diagnose MI based on the level of D-dimer, the rock curve was used and to calculate the sensitivity, specificity and positive and negative predictive value of D-dimer in the diagnosis of MI, MedCalc 15 software was used.

\section{Results}

\section{Baseline characteristics}

Over the 10-month course of study, 97 patients were enrolled and 22 patients were excluded. Exclusion causes included a positive family history of cardiovascular disease (14 patients), using heart medications like beta blockers, nitrate and aspirin (four patients), occurrence of arrhythmias (two patients), and using anticoagulants (two patients). Finally, 75 patients with a mean age of $63.1 \pm 9.75$ years were studied. The two groups 
Table 2. Comparing Serum Level of D-Dimer and Troponin in Three Groups of Patients With Myocardial Infarction With ST Segment Elevation, Without ST Segment Elevation, and Unstable Angina

\begin{tabular}{|c|c|c|c|c|}
\hline Variables & $\begin{array}{l}\text { Myocardial infarction with } \\
\text { ST segment elevation }(n=34)\end{array}$ & $\begin{array}{l}\text { Myocardial infarction without } \\
\text { ST segment elevation }(n=12)\end{array}$ & Unstable angina $(n=29)$ & $\mathbf{P}$ \\
\hline Troponin (mean $\pm \mathrm{SD}), \mathrm{mg} / \mathrm{dL}$ & $0.73 \pm 0.4$ & $0.51 \pm 0.3$ & $0.02 \pm 0.1$ & 0.001 \\
\hline
\end{tabular}

of patients with MI $(\mathrm{n}=34)$ and UA $(\mathrm{n}=41)$ were not significantly different in age, gender, and risk factors for diabetes and dyslipidemia (Table 1).

\section{Troponin and D-dimer level}

Average troponin in all patients was $0.42 \pm 0.4 \mathrm{mEq} / \mathrm{L}$. MI group has a higher level of serum troponin in admission compared to UA group ( $\mathrm{P}=0.001)$ (Table 1). Besides, the mean serum level of D-dimer was $1,087.61 \pm 395.63 \mathrm{mEq} / \mathrm{L}$ in all patients and was higher in patients with MI than in patients with UA $(\mathrm{P}=0.001)$ (Table 1$)$.

\section{Subgroup analysis}

Moreover, the results of this study showed that the three groups of STEMI, NSTEMI and UA are significantly different in terms of troponin levels $(\mathrm{P}=0.001)$ and $\mathrm{D}$-dimer levels $(\mathrm{P}=$ 0.001), based on ANOVA (Table 2). But according to the post hoc test, this dispute arises from the difference in the serum levels between the group of patients with UA and NSTEMI and the group with UA and STEMI (Table 2).

\section{D-dimer and correlated factors}

Based on the analysis of Pearson correlation, a moderate to weak correlation was observed between $\mathrm{D}$-dimer and age $(\mathrm{P}=$ $0.005, \mathrm{r}=0.32)$, blood glucose $(\mathrm{P}=0.006, \mathrm{r}=0.31)$ and troponin $(\mathrm{P}=0.000, \mathrm{r}=0.57)$.

\section{Diagnostic value of D-dimer}

To find the cut-off point with the highest sensitivity and specificity of D-dimer serum level in the diagnosis of MI, ROC curve was used. Based on the results of ROC curve, the best cut-off point for $\mathrm{MI}$ is $548 \mathrm{mEq} / \mathrm{L}$ in terms of D-dimer (Fig. $1)$. The sensitivity and specificity of this point in differentiating MI from UA in patients presenting with ACS is $63.4 \%$ and

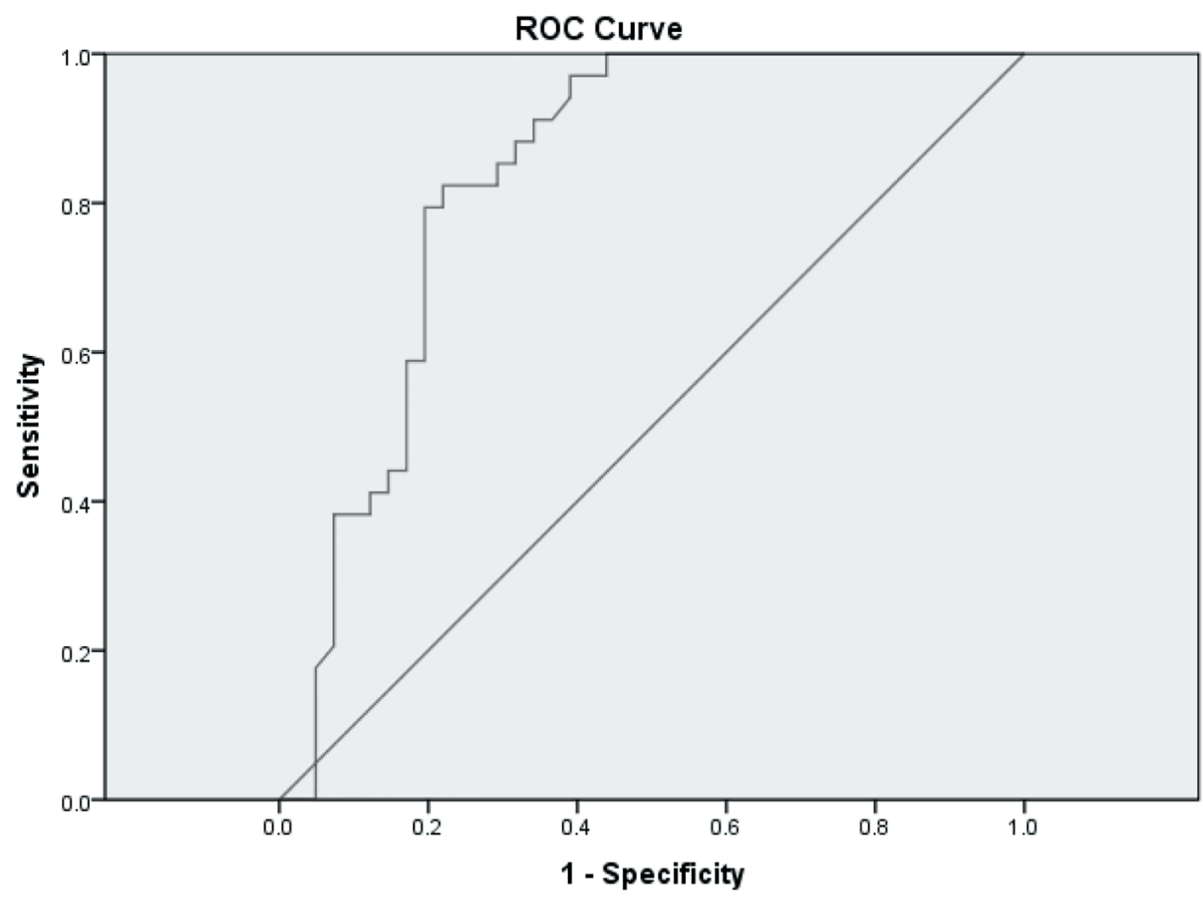

Diagonal segments are produced by ties.

Figure 1. Receiver operating characteristic curve on the diagnostic value of D-dimer in diagnosis of acute myocardial infarction. 
Table 3. Operative Characteristics of D-Dimer for the Diagnosis of Myocardial Infarction

\begin{tabular}{lll}
\hline Discriminate level (ng/mL) & Sensitivity, \% & Specificity, \% \\
\hline 474 & 100 & 56.1 \\
548 & 91.2 & 63.4 \\
682 & 85.3 & 70.7 \\
548 & 91.2 & 63.4 \\
\hline
\end{tabular}

$91.2 \%$, respectively (Table 3 ).

\section{Discussion}

In the past, D-dimer was simply used as a marker in venous thromboembolism as well as aortic dissection and few studies have been conducted on its role in the diagnosis of MI [1-5]. Our study was aimed to determine the diagnostic value of a D-dimer test for MI in patients with suspected ACS and the results showed that $\mathrm{D}$-dimer can also be used as a diagnostic marker with appropriate sensitivity and specificity in the diagnosis of MI.

Today, the measurement of troponin is considered as one of the most selective markers in the diagnosis of myocardial damage $[5,8]$. Although, the fundamental flaw against them is an increase in their serum level $3-4 \mathrm{~h}$ after the onset of symptoms. Thus, in most centers, these markers should be reviewed in consecutive times. And in this case, their application in rapid triage of patients with MI is faced with ambiguity [2]. Also, it is still believed that more biomarkers should be found for the faster diagnosis of coronary thrombosis as a hallmark of ACS [1]. D-dimer is produced by destruction and the breakdown of fibrin clot at the site of injury by plasmin, which represents the manufacture of active thrombosis and its lysis [11, 12]. D-dimer level is expected to increase in acute ischemic events faster than in other cardiac markers because D-dimer is created faster than other markers in the course of the ACS pathophysiology $[9,12]$.

On the other hand, studies have shown that D-dimer is in direct relationship with the occurrence or recurrence of cardiovascular diseases, so that patients whose D-dimer is in upper one-third of D-dimer levels, are 70\% more at risk of CHD than those in lower one-third of D-dimer levels [3]. Baya-Genis et al have shown that plasma level of D-dimer in patients with $\mathrm{MI}$ and UA is more than in patients without ischemic event. Also, plasma D-dimer in patients with MI was higher than in patients with UA [3]. Besides, this study also demonstrated that D-dimer greater than $500 \mathrm{ng} / \mathrm{mL}$ along with ECG findings and patients' histories, can improve diagnosis of MI (sensitivity increases from $73 \%$ to $92 \%$ ) [3]. In our study, the best cutoff point was achieved in differentiating MI from UA in 548, so that it had sensitivity of $91.2 \%$ and specificity of $63.4 \%$. Moreover, by raising the cut-off point to $682 \mathrm{ng} / \mathrm{mL}$ in our study, the results indicate an increase in specificity of D-dimer test up to $70.7 \%$ and by lowering the cut-off point to 474 $\mathrm{ng} / \mathrm{mL}$, the results indicate an increase in D-dimer sensitivity up to $100 \%$. Considering the present results of this study and
Bayes-Genis' study, it seems that the serum level higher than $500-550 \mathrm{ng} / \mathrm{mL}$ can be assumed as a reliable cut-off point in differentiating MI from UA in reality as well [3]. The main objective of our study is differentiating MI from non-MI in patients with ACS. However, most studies have evaluated the diagnostic value of $\mathrm{D}$-dimer in forecasting ACS from pains with non-cardiac origin. In Orak et al's study, D-dimer serum level in the case group with ACS was obtained about $2.31 \pm$ 2.34 and the D-dimer sensitivity and specificity in the differentiating ACS from non-ACS was specified as $95.4 \%$ and $83.7 \%$, respectively [13]. However, in all studies, high positive predictive value of D-dimer has not been reported. And it is expressed in Lippi et al's study that D-dimer cannot be used as an independent diagnostic factor in MI due to specificity and low negative predictive value ( $41 \%$ and $27 \%$, respectively) and it must be used alongside another diagnostic marker [10]. Moreover, the use of multiple biomarkers including D-dimer has been recommended in Tello-Montoliu's study in diagnosis of ACS [4]. But what seems to be the main cause of the difference between the results such as the found cut-off point and the diagnostic power of that point for diagnosis of MI or ACS, is the duration of onset of symptoms up to measuring D-dimer level apart from racial differences, sample size, the instrument used to measure D-dimer and the applied method and this is the subject which has not been referred to in various studies including ours and is one of the limitations of our study. Besides, other limitations of this study are the lack of a control group of normal subjects. If a group of normal subjects were enrolled in this study, we could evaluate the diagnostic power of Ddimer in differentiating ACS from non-ACS events. Moreover, a recent study has not examined the prognostic value of Ddimer in patients with ACS, while some studies conducted in recent decade have examined the prognostic value of D-dimer in predicting mortality following ACS [7, 14-16]. Therefore, it is recommended that future studies to be carried out as clinical trials for comparing different methods of measuring D-dimer and its impact on its diagnostic power in differentiating ACS from non-ACS and MI from UA.

\section{Conclusion}

Based on the results of this study, it seems that the measurement of D-dimer serum level can be appropriate as a marker with high sensitivity and relatively high specificity for differentiating MI from UA in patients with suspected ACS. Especially, given the high sensitivity of this marker, and its availability in most EDs, it seems that D-dimer can be used for the appropriate triage of patients, and referring them to cardiologists fast for more specialized treatments. However, it is suggested other cardiac biomarkers to be used in combination with D-dimer measurement based on the findings of other results in order to improve diagnostic power of D-dimer.

\section{Conflict of Interest}

The author declare that there is no conflict of interest regarding 
the publication of this paper.

\section{References}

1. Wu AH, Smith A, Christenson RH, Murakami MM, Apple FS. Evaluation of a point-of-care assay for cardiac markers for patients suspected of acute myocardial infarction. Clin Chim Acta. 2004;346(2):211-219.

2. $\mathrm{Wu} \mathrm{AH}$, Christenson RH. Analytical and assay issues for use of cardiac troponin testing for risk stratification in primary care. Clin Biochem. 2013;46(12):969-978.

3. Bayes-Genis A, Mateo J, Santalo M, Oliver A, Guindo J, Badimon L, Martinez-Rubio A, et al. D-Dimer is an early diagnostic marker of coronary ischemia in patients with chest pain. Am Heart J. 2000;140(3):379-384.

4. Tello-Montoliu A, Marin F, Roldan V, Mainar L, Lopez MT, Sogorb F, Vicente V, et al. A multimarker risk stratification approach to non-ST elevation acute coronary syndrome: implications of troponin T, CRP, NT pro-BNP and fibrin D-dimer levels. J Intern Med. 2007;262(6):651658.

5. Fathil MF, Md Arshad MK, Gopinath SC, Hashim U, Adzhri R, Ayub RM, Ruslinda AR, et al. Diagnostics on acute myocardial infarction: Cardiac troponin biomarkers. Biosens Bioelectron. 2015;70:209-220.

6. Bergh TH, Steen K, Lindau T, Soldal LA, Bernardshaw SV, Lunde L, Lie SA, et al. Costs analysis and comparison of usefulness of acute MRI and 2 weeks of cast immobilization for clinically suspected scaphoid fractures. Acta Orthop. 2015;86(3):303-309.

7. Danesh J, Whincup P, Walker M, Lennon L, Thomson A, Appleby P, Rumley A, et al. Fibrin D-dimer and coronary heart disease: prospective study and meta-analysis. Circulation. 2001;103(19):2323-2327.

8. Hamm CW, Goldmann BU, Heeschen C, Kreymann $\mathrm{G}$, Berger J, Meinertz T. Emergency room triage of patients with acute chest pain by means of rapid testing for cardiac troponin T or troponin I. N Engl J Med. 1997;337(23):1648-1653.

9. Kruskal JB, Commerford PJ, Franks JJ, Kirsch RE. Fibrin and fibrinogen-related antigens in patients with stable and unstable coronary artery disease. N Engl J Med. 1987;317(22):1361-1365.

10. Lippi G, Filippozzi L, Montagnana M, Salvagno GL, Guidi GC. Diagnostic value of D-dimer measurement in patients referred to the emergency department with suspected myocardial ischemia. J Thromb Thrombolysis. 2008;25(3):247-250.

11. Wada H, Sakuragawa N. Are fibrin-related markers useful for the diagnosis of thrombosis? Semin Thromb Hemost. 2008;34(1):33-38.

12. Righini M, Robert-Ebadi H, Le Gal G. Diagnosis of pulmonary embolism. Presse Med. 2015;44(12 Pt 2):e385391.

13. Orak M, Ustundag M, Guloglu C, Alyan O, Sayhan MB. The role of serum D-dimer level in the diagnosis of patients admitted to the emergency department complaining of chest pain. J Int Med Res. 2010;38(5):1772-1779.

14. Ridker PM, Hennekens CH, Cerskus A, Stampfer MJ. Plasma concentration of cross-linked fibrin degradation product (D-dimer) and the risk of future myocardial infarction among apparently healthy men. Circulation. 1994;90(5):2236-2240.

15. Menown IB, Mathew TP, Gracey HM, Nesbitt GS, Murray P, Young IS, Adgey AA, et al. Prediction of recurrent events by D-Dimer and inflammatory markers in patients with normal cardiac Troponin I (PREDICT) Study. Am Heart J. 2003;145(6):986-992.

16. Lowe GD, Yarnell JW, Sweetnam PM, Rumley A, Thomas HF, Elwood PC. Fibrin D-dimer, tissue plasminogen activator, plasminogen activator inhibitor, and the risk of major ischaemic heart disease in the Caerphilly Study. Thromb Haemost. 1998;79(1):129-133. 